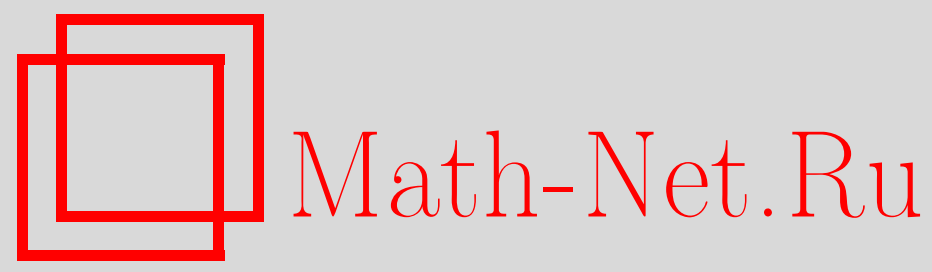

А. П. Розовская, Д. А. Шабанов, Экстремальные задачи для полноцветных раскрасок равномерных гиперграфов, Дискрет. матем., 2012, том 24, выпуск 2, 104-122

DOI: https://doi.org/10.4213/dm1188

Использование Общероссийского математического портала Math-Net.Ru подразумевает, что вы прочитали и согласны с пользовательским соглашением http://www . mathnet.ru/rus/agreement

Параметры загрузки:

IP : 18.234 .156 .22

26 апреля 2023 г., 15:14:37 


\title{
Экстремальные задачи для полноцветных раскрасок равномерных гиперграфов
}

\author{
() 2012 г. А. П. Розовская, Д. А. Шабанов
}

В работе исследуется известная задача экстремальной теории гиперграфов, поставленная А. В. Косточкой. Раскраска множества вершин гиперграфа в $r$ цветов называется полноцветной, если в ней каждое ребро гиперграфа содержит вершины всех цветов. Изучается величина $p(n, r)$, равная минимально возможному количеству ребер $n$-равномерного гиперграфа, не имеющего полноцветных $r$-раскрасок. В работе найдена новая нижняя асимптотическая оценка величины $p(n, r)$, а также получен ряд результатов в смежных задачах.

Работа выполнена при поддержке Российского фонда фундаментальных исследований, грант 12-01-00683a, Программы Президента РФ поддержки молодых российских ученых, грант МК 1122.2012.1, и Программы Президента РФ поддержки ведущих научных школ России, грант НШ 2519.2012.1.

\section{1. Введение и история задачи}

В работе исследуется известная задача экстремальной теории гиперграфов. Напомним, что гиперграфом называется пара множеств $H=(V, E)$, где $V=V(H)$ есть некоторое конечное множество, называемое множеством вершин гиперграфа, а $E=E(H)$ есть совокупность некоторых подмножеств множества $V$, эти подмножества называются ребрами гиперграфа. Говорим, что гиперграф является $n$-равномерным, если каждое его ребро содержит ровно $n$ вершин.

Раскраска множества вершин гиперграфа в $r$ цветов ( $r$-раскраска) называется полноцветной для $H$, если в ней каждое ребро из $E$ содержит вершины всех цветов. В работе А. В. Косточки [1] (см. также [2]) была поставлена задача об отыскании величины $p(n, r)$, равной минимальному числу ребер гиперграфа в классе $n$-равномерных гиперграфов, не имеющих полноцветных $r$-раскрасок. Формально определение $p(n, r)$ можно записать так:

$$
p(n, r)=\min \{|E(H)|: H \text { есть } n \text {-равномерный гиперграф, }
$$

для которого не существует полноцветных $r$-раскрасок\}.

Задача о нахождении величины $p(n, r)$ хорошо изучена в случае $r=2$. Величина $p(n, 2)$ совпадает с классической величиной $m(n)$, равной минимальному количеству ребер $n$-равномерного гиперграфа, вершины которого нельзя покрасить в два цвета так, чтобы все ребра были неодноцветны. Задача об отыскании величины $m(n)$ была поставлена в 1961 году П. Эрдёшем и А. Хайналом в [3]. Наилучшие асимптотические оценки 
величины $m(n)$ были получены Эрдёшем (верхняя оценка, [4]), а также Дж. Радхакришнаном и А. Сринивасаном (нижняя оценка, [5]):

$$
(\sqrt{3}-1)\left(\frac{n}{\ln n}\right)^{1 / 2} 2^{n-1} \leqslant p(n, 2)=m(n) \leqslant \frac{e \ln 2}{4} n^{2} 2^{n}(1+o(1)) .
$$

Одно из первых достаточных условий существования полноцветной $r$-раскраски в общем случае было получено П. Эрдёшем и Л. Ловасом в известной работе [6]: если каждое ребро $n$-равномерного гиперграфа $H$ пересекается не более чем с $r^{n-1} /\left(4(r-1)^{n}\right)$ другими ребрами, то для $H$ существует полноцветная $r$-раскраска. Таким образом, фактически была получена следующая нижняя оценка величины $p(n, r)$ :

$$
p(n, r) \geqslant \frac{r^{n-1}}{4(r-1)^{n}} .
$$

Общие соотношения для произвольного $r$ были получены А. В. Косточкой. С использованием связи между полноцветными $r$-раскрасками $n$-равномерных гиперграфов и предписанными раскрасками полных $r$-дольных графов, в [1]) были получены следующие оценки для $p(n, r)$ при $r \geqslant 2$ и $n \geqslant 2$ :

$$
\frac{1}{r} e^{c_{1} n / r} \leqslant p(n, r) \leqslant r e^{c_{2} n / r},
$$

где $c_{1}$ и $c_{2}$ - некоторые абсолютные положительные константы, причем $c_{1}<1<c_{2}$.

В $[7,8]$ Д. А. Шабановым были получены следующие оценки $p(n, r)$ для случая $r=3$ :

$$
c\left(\frac{n}{\ln n}\right)^{1 / 3}\left(\frac{3}{2}\right)^{n} \leqslant p(n, 3) \leqslant \frac{e \ln 3}{12} n^{2}\left(\frac{3}{2}\right)^{n}(1+o(1)),
$$

где $c$ - некоторая положительная константа. Наконец, в [9] были получены аналогичные оценки величины $p(n, r)$ в общем случае. Нижняя оценка справедлива для всех $n, r \geqslant 2$, $r<n$, и имеет вид:

$$
p(n, r) \geqslant \frac{\sqrt{21}-3}{4 r}\left(\frac{n}{(r-1)^{2} \ln n}\right)^{1 / 3}\left(\frac{r}{r-1}\right)^{n} .
$$

Верхняя оценка в [9] была получена в двух различных областях значений функции $r=r(n)$. Если $r=r(n)=o(\sqrt{n})$, то

$$
p(n, r) \leqslant \frac{1}{r}\left(\frac{r}{r-1}\right)^{n} e(\ln r) \frac{n^{2}}{2(r-1)} \varphi_{1},
$$

где $\varphi_{1}-$ некоторая функция от $n$ и $r(n)$, стремящаяся к единице при $n \rightarrow \infty$. Если же $\sqrt{n} \ll r<\frac{1}{2} n^{2 / 3}$ (то есть $n=o\left(r^{2}\right)$ ), то

$$
p(n, r) \leqslant \frac{1}{r}\left(\frac{r}{r-1}\right)^{n} e(\ln r) n^{3 / 2} \varphi_{2}
$$

где $\varphi_{2}$ - некоторая функция от $n$ и $r(n)$, стремящаяся к единице при $n \rightarrow \infty$.

До настоящего исследования, результаты (1), (4), (5) и (6) оставались наилучшими в различных областях значений параметров. 


\section{2. Новая нижняя оценка $p(n, r)$}

Одним из основных результатов данной работы является новая нижняя оценка величины $p(n, r)$.

Теорема 1. Для любых $n \geqslant 3, r \geqslant 2$ с условием $r<n /(2 \ln n)$ верно, что

$$
p(n, r) \geqslant \frac{1}{2 r}\left(\frac{n}{(r-1)^{2} \ln n}\right)^{1 / 2}\left(\frac{r}{r-1}\right)^{n} .
$$

Нетрудно видеть, что данный результат улучшает предыдущие результаты (2), (3) и (4) в области $r=o(\sqrt{n / \ln n})$. Действительно, улучшение по сравнению с классической оценкой Эрдёша и Ловаса (2), а также с оценкой Косточки (3) очевидно. Кроме того, оценка (7) асимптотически улучшает и (4) в указанной области. При этом асимптотическое улучшение имеет порядок $\left(n /\left(r^{2} \ln n\right)\right)^{1 / 6}$. Отметим также, что при $r=2$ результат (7) совпадает по порядку с наилучшей нижней оценкой (1) для величины $m(n)=p(n, 2)$, а зазор между (7) и верхней оценкой (5) имеет порядок $n^{3 / 2}(\ln n)^{1 / 2}$, как и для случая $r=2$.

Полноцветные раскраски гиперграфов тесно связаны с предписанными раскрасками полных многодольных графов. Обсудим подробнее эту связь, но сначала напомним ряд определений.

Пусть $G=(V, E)$ - обыкновенный граф с множеством вершин $V$ и множеством ребер $E$, а $C$ - некоторое множество, элементы которого назовем цветами. Вершинным предписанием $A$ будем называть некоторое отображение, которое каждой вершине $v$ сопоставляет в соответствие некоторое подмножество $A(v) \subseteq C$. Если $|A(v)|=k$ для любой вершины $v \in V$, то будем говорить, что мощность предписания равна $k$.

Раскраской $f$ вершин графа $G$, соответствующей предписанию $A$, называется такое отображение $f: V \rightarrow C$, что $f(v) \in A(v)$. При этом будем говорить, что каждая вершина $v$ окрашена в цвет $f(v)$. Раскраска множества вершин называется правильной для графа $G$, если любые две вершины $G$, соединенные ребром, окрашены в разные цвета. Граф называется предписанно $k$-раскрашиваемым, если для любого множества цветов $C$ и любого вершинного предписания $A$ мощности $k$ найдется правильная раскраска, соответствующая данному предписанию. Предписанным хроматическим числом графа $G$ называется минимальное натуральное число $k$ такое, что $G$ является предписанно $k$-раскрашиваемым.

Изучение предписанного хроматического числа было начато в работах В. Г. Визинга [10], а также П. Эрдёша, А. Л. Рубина и Х. Тейлора [11]. В [11] была поставлена задача об отыскании величины $N(r, n)$ - минимального числа вершин в полном $r$-дольном графе, предписанное хроматическое число которого превышает $n$. Эрдешём, Рубином и Тейлором была показана (см. [11]) тесная связь между $N(2, n)$ и классической величины $m(n)$ : именно, имеет место соотношение

$$
m(n) \leqslant N(2, n) \leqslant 2 m(n) .
$$

А. В. Косточка в [1] обобщил данное неравенство на случай произвольного числа $r$ :

$$
p(n, r) \leqslant N(r, n) \leqslant r p(n, r) .
$$

Из неравенств, полученных Н. Алоном (см. [12]) для предписанных хроматических чисел полных $r$-дольных графов, вытекают следующие оценки величины $N(r, n)$ :

$$
\exp \left\{c_{1} \frac{n}{r}\right\} \leqslant N(r, n) \leqslant \exp \left\{c_{2} \frac{n}{r}\right\}
$$


где $c_{1}, c_{2}-$ те же константы, что и в соотношениях (3).

Позднее обе оценки $N(r, n)$ были улучшены Шабановым в [9] с помощью оценок (4), (5) и (6) величины $p(n, r)$. В частности, было доказано, что для любых $r \geqslant 2, n \geqslant 3$ выполняется неравенство

$$
N(r, n) \geqslant \frac{\sqrt{21}-3}{4 r}\left(\frac{n}{(r-1)^{2} \ln n}\right)^{1 / 3}\left(\frac{r}{r-1}\right)^{n}
$$

Оценка (10) асимптотически улучшает результат (9) при $r=r(n)=o(\sqrt{n / \ln n})$. Теорема 1 с помощью неравенств (8) позволяет получить новую нижнюю оценку величины $N(r, n)$ при условии $r<n /(2 \ln n)$ :

$$
N(r, n) \geqslant \frac{1}{2 r}\left(\frac{n}{(r-1)^{2} \ln n}\right)^{1 / 2}\left(\frac{r}{r-1}\right)^{n} .
$$

Наша новая оценка (11), как нетрудно видеть, асимптотически улучшает (10) в области $r=o(\sqrt{n / \ln n})$.

В заключение отметим, что с помощью исследования асимптотического поведения величины $p(n, r)$ оказывается возможным получить асимптотику предписанного хроматического числа полных $r$-дольных графов с одинаковым размером долей. Подробнее об этом можно прочитать в [13], а в следующем разделе мы докажем основную теорему 1.

\section{3. Доказательство теоремы 1}

Пусть заданы $n \geqslant 3, r \geqslant 2$ и $r \leqslant n /(2 \ln n)$. Для доказательства теоремы достаточно показать, что для произвольного $n$-равномерного гиперграфа $G=(V, E)$ с условием

$$
|E|<\frac{1}{2 r}\left(\frac{n}{(r-1)^{2} \ln n}\right)^{1 / 2}\left(\frac{r}{r-1}\right)^{n}
$$

существует полноцветная раскраска множества $V$ в $r$ цветов.

Зафиксируем такой гиперграф $G$. Пусть задана произвольная раскраска множества вершин $V$ в $r$ цветов. Будем называть ребро хорошим в данной раскраске, если в нем присутствуют вершины каждого из $r$ цветов. Иначе будем говорить, что ребро плохое в этой раскраске. Докажем, что для гиперграфа $G$ найдется раскраска, в которой все его ребра будут хорошими. Для этого построим некоторую случайную раскраску и оценим вероятность того, что в ней есть плохие ребра.

\section{1. Алгоритм построения случайной раскраски}

Пусть $\sigma$ - случайная нумерация множества вершин гиперграфа с равномерным распределением. Раскрасим каждую вершину гиперграфа независимо от других в один из $r$ цветов с вероятностью $1 / r$. Обозначим получившуюся равномерную раскраску через $\xi$. Построим на ее основе другую раскраску. Применим следующий алгоритм перекраски.

(1) Рассматриваем вершины $G$ по очереди, согласно нумерации $\sigma$.

(2) На $i$-м шаге процедуры перекраски рассматриваем вершину $v$, такую, что $\sigma(v)=i$. Предположим, что для вершины $v$ выполнены оба следующих условия: 
(a) вершина $v$ принадлежит ребру $e$, которое содержит вершины ровно $r-1$ цвета в раскраске $\xi$ (пусть, без ограничения общности, отсутствует цвет $\alpha$ ),

(b) ни на одном из предыдущих шагов перекраски ни одна из вершин ребра $e$ не перекрасилась в цвет $\alpha$.

Тогда случайно перекрашиваем вершину $v$ согласно следующему правилу: с вероятностью $p$ присваиваем каждый из $r-1$ цветов, отличных от начального цвета $v$, и с вероятностью $1-(r-1) p$ вообще не перекрашиваем вершину $v$.

Если же хотя бы одно из перечисленных выше условий не выполнено, то мы также не перекрашиваем $v$ и переходим к рассмотрению следующей вершины.

Обозначим получившуюся после рассмотрения всех вершин $G$ случайную раскраску через $\chi$.

Обозначим через $\mathscr{F}_{e}$ событие, заключающееся в том, что ребро $e$ плохое в раскраске $\chi$. Чтобы оценить вероятность этого события, разобьем его в сумму более простых событий. Рассмотрим ситуации, в которых ребро $e$ может оказаться плохим в раскраске $\chi$.

(1) Ребро $e$ в раскраске $\xi$ содержит вершины не более чем $r-2$ различных цветов. Обозначим это событие через $A_{e}^{1}$.

(2) Ребро $e$ в раскраске $\xi$ содержит вершины ровно $r-1$ различных цветов и после перекраски в нем не появился недостающий последний цвет. Обозначим это событие через $A_{e}^{2}$.

(3) Ребро $е$ в раскраске $\xi$ содержит вершины ровно $r-1$ цветов, в процессе перекраски в нем появился недостающий последний цвет, но к моменту его появления исчез один из других $r-1$ цветов. Обозначим это событие через $A_{e}^{3}$.

(4) Ребро $е$ является плохим в итоговой раскраске $\chi$, но не случилась ни одна из предыдущих ситуаций. Здесь возможны два варианта.

(а) Ребро $e$ в раскраске $\xi$ содержит вершины ровно $r-1$ цветов, в процессе перекраски в нем появился недостающий последний цвет, и в этот момент оно стало хорошим, но в дальнейшем ребро $e$ стало плохим в результате перекрашивания некоторых его вершин, принадлежащих некоторому другому ребру $f$, которое также являлось плохим в $\xi$.

(b) Ребро $e$ является хорошим в раскраске $\xi$ и плохим в итоговой раскраске, то есть оно испортилось в результате перекрашивания некоторых его вершин, принадлежащих некоторому другому ребру $f$, которое являлось плохим в $\xi$.

Обе приведенные ситуации можно обобщить следующим образом. В ребре $e$ были вершины некоторого цвета $\alpha$ в раскраске $\xi$, но в раскраске $\chi$ в $e$ нет вершин этого цвета, причем последняя из них перекрасилась вследствие того, что принадлежало некоторому другому плохому ребру. Если $v$ - последняя (относительно случайной нумерации $\sigma$ ) вершина ребра $e$, которая покрашена в цвет $\alpha$ в раскраске $\xi$, тогда существует некоторое ребро $f, v \in f$, которое было покрашено ровно в $r-1$ цвет в $\xi$, и до момента рассмотрения $v$ этот недостающий цвет в ребре $f$ не появился. Обозначим данное событие через $B_{e, f}$. 
Подведем предварительные итоги. Мы показали, что для каждого ребра $e$ событие $\mathscr{F} e$ лежит в объединении

$$
\mathscr{F}_{e} \subset A_{e}^{1} \cup A_{e}^{2} \cup A_{e}^{3} \cup \bigcup_{f \neq e} B_{e, f} .
$$

Далее мы оценим вероятности этих событий.

\section{2. Вероятностная интерпретация}

В предыдущем разделе мы привели алгоритм построения правильной раскраски вершин гиперграфа. Однако в дальнейших доказательствах нам понадобится определение случайной раскраски в терминах случайных величин. Действительно, раскраску $\xi$ множества вершин гиперграфа $V$ (без ограничения общности считаем, что $V=\{1, \ldots, w\})$ можно рассматривать как набор независимых одинаково распределенных случайных величин $\xi_{1}, \ldots, \xi_{w}$, принимающих значения $1, \ldots, r$ с одной и той же вероятностью, равной $1 / r$. Введем набор одинаково распределенных случайных величин $\eta_{1}, \ldots, \eta_{w}$, распределение которых задается следующими условными вероятностями:

$$
\mathbf{P}\left(\eta_{i}=k \mid \xi_{i}=j\right)=p, \quad j \neq k, \quad j=1, \ldots, r
$$

и

$$
\mathbf{P}\left(\eta_{i}=0 \mid \xi_{i}=j\right)=1-(r-1) p .
$$

Кроме того, будем считать, что случайная нумерация $\sigma$ не зависит от набора $\left\{\xi_{1}, \ldots, \xi_{w}\right\}$. Саму нумерацию $\sigma$ можно также рассматривать как набор случайных величин $\sigma_{1}, \ldots, \sigma_{w}$.

Пусть $i \in V-$ произвольная вершина гиперграфа. Обозначим через $D_{i}$ событие, заключающееся в том, что вершину $i$ следует перекрашивать:

$$
\begin{aligned}
D_{i}=\bigcup_{\substack{e \in E: j \\
i \in e}} \bigcup_{j=1}^{r}\left(\left\{\sum_{s \in e} I\left\{\xi_{s} \neq j\right\}\left(1-I\left\{\sigma_{s}<\sigma_{i}, \eta_{s}=j\right\}\right)=n\right\}\right. \\
\left.\cap\left\{\bigcap_{\substack{k=1 \\
k \neq j}}^{r}\left(\sum_{s \in e} I\left\{\xi_{s}=k\right\}>0\right)\right\}\right) .
\end{aligned}
$$

Тогда раскраска $\chi$ есть набор случайных величин $\chi_{1}, \ldots, \chi_{w}$, задаваемых равенством

$$
\chi_{i}=\xi_{i} I\left\{\bar{D}_{i} \cup\left\{\eta_{i}=0\right\}\right\}+\eta_{i} I\left\{D_{i} \cap\left\{\eta_{i} \neq 0\right\}\right\},
$$

а интересующие нас события записываются следующим образом:

$$
\begin{aligned}
A_{e}^{1} & =\bigcup_{\alpha, \beta=1}^{r} \bigcap_{s \in e}\left\{\xi_{s} \neq \alpha, \xi_{s} \neq \beta\right\}=\tilde{A}_{e}^{1}, \\
A_{e}^{2} & \left.=\bigcup_{\alpha=1}^{r} \bigcap_{s \in e}\left\{\left\{\xi_{s} \neq \alpha, \chi_{s} \neq \alpha\right\} \cap\left\{\bigcap_{\substack{k=1 \\
k \neq j}}^{r}\left(\sum_{s \in e} I\left\{\xi_{s}=k\right\}>0\right)\right\}\right)\right) \\
& \subseteq \bigcup_{\alpha=1}^{r}\left\{\begin{array}{l}
\left\{\xi_{s} \neq \alpha, \eta_{s} \neq \alpha\right\}=\tilde{A}_{e}^{2},
\end{array}\right.
\end{aligned}
$$




$$
\begin{aligned}
A_{e}^{3} \subseteq \bigcup_{\substack{\alpha \neq \beta \\
\alpha, \beta=1}}^{r} \bigcup_{u \in e} \bigcap_{s \in e}\left(\left\{\sigma_{s}>\sigma_{u}, \xi_{s} \neq \alpha, \beta\right\}\right. \\
\cup\left\{\sigma_{s}<\sigma_{u}, \xi_{s}=\beta, \eta_{s} \notin\{0, \beta, \alpha\}\right\} \\
\cup\left\{\sigma_{s}<\sigma_{u}, \xi_{s} \neq \alpha, \beta, \eta_{s} \neq \alpha, \beta\right\} \\
\left.\cup\left\{\xi_{u} \neq \alpha, \eta_{u}=\alpha\right\}\right)=\tilde{A}_{e}^{3} .
\end{aligned}
$$

Поясним данное соотношение. Пусть в ребре $e$ не было цвета $\alpha$ в раскраске $\xi$, а в процессе перекраски из него исчез цвет $\beta$. Пусть $u$ - первая (согласно нумерации $\sigma)$ вершина в ребре $e$, перекрасившаяся в цвет $\alpha$. Если произошло событие $A_{e}^{3}$, то все вершины, следующие за $u$ по нумерации $\sigma$, должны быть окрашены не в цвет $\alpha$ и не в цвет $\beta$ в начальной раскраске $\xi$. Вершины, идущие по нумерации до $u$, либо имеют цвет $\beta$ и перекрашиваются в один из $r-2$ цветов (исключая цвет $\alpha$ ), либо имеют цвет, отличный от $\alpha$ и $\beta$, в обеих раскрасках.

Рассмотрим теперь событие $B_{e, f}$. Верно соотношение

$$
\begin{aligned}
B_{e, f} \subseteq & \bigcup_{\alpha, \gamma=1}^{r} \bigcup_{v \in e \cap f}\left(\left\{\xi_{v}=\alpha, \eta_{v} \notin\{0, \alpha\}\right\} \cap \bigcap_{s \in f \backslash\{v\}}\left\{\xi_{s} \neq \gamma\right\}\right. \\
& \cap \bigcap_{s \in f \backslash e}\left[\left\{\sigma_{s}<\sigma_{v}, \eta_{s} \neq \gamma\right\} \cup\left\{\sigma_{s}>\sigma_{v}\right\}\right] \\
& \cap \bigcap_{s \in e}\left[\left\{\sigma_{s}>\sigma_{v}, \xi_{s} \neq \alpha\right\} \cup\left\{\sigma_{s}<\sigma_{v}, \xi_{s} \neq \alpha\right\}\right. \\
& \left.\cap \bigcap_{s \in e \cap f} \cup\left\{\sigma_{s}<\sigma_{v}, \xi_{s}=\alpha, \eta_{s} \notin\{0, \alpha\}\right\}\right] \\
& \left.\left(\left\{\sigma_{s}<\sigma_{v}, \xi_{s} \neq \gamma, \sigma_{v}, \xi_{s}=\alpha, \eta_{s} \neq \gamma\right\} \cup\left\{\sigma_{s}>\sigma_{v}, \xi_{s} \neq \gamma, \xi_{s} \neq \alpha\right\}\right)\right) \\
= & \widetilde{B}_{e, f},
\end{aligned}
$$

где $v$ - некоторая вершина из $e \cap f$. Поясним данное соотношение. Действительно, пусть выполнено событие $B_{e, f}$. Тогда в ребре $e$ в процессе перекраски исчезли вершины некоторого цвета $\alpha$. Пусть $v$ - последняя по нумерации $\sigma$ вершина цвета $\alpha$ в $e$ в раскраске $\xi$, тогда по определению события $B_{e, f}$ найдется ребро $f$, окрашенное в $(r-1)$-й цвет в раскраске $\xi$ (пусть в нем отсутствует цвет $\gamma \neq \alpha$ ), такое, что $v \in e \cap f$. Кроме того, ни одна вершина, предшествующая $v$ по нумерации $\sigma$ в ребре $f$, не перекрасилась в цвет $\gamma$, и вершина $v$ поменяла свой цвет. Также все вершины ребра $e$ не должны быть окрашены в цвет $\alpha$ в итоговой раскраске $\chi$. При этом все вершины ребра $e$, идущие по нумерации после вершины $v$, не окрашены в цвет $\alpha$ уже в раскраске $\xi$. Если же для вершины $u \in e$ выполнено $\sigma(u)<\sigma(v)$, то либо $\xi_{u} \neq \alpha$, либо вершина $u$ имеет цвет $\alpha$ в раскраске $\xi$, но перекрасилась в другой цвет (то есть $\xi_{u}=\alpha, \eta_{u} \neq 0$ ).

Перейдем теперь непосредственно к оцениванию вероятностей указанных событий.

\section{3. Оценки вероятностей событий}

Разобьем оценки вероятностей описанных событий на несколько лемм. 
Лемма 1. Верны неравенства

$$
\begin{aligned}
& \mathbf{P}\left(A_{e}^{1}\right) \leqslant \mathbf{P}\left(\tilde{A}_{e}^{1}\right)=\left(\begin{array}{l}
r \\
2
\end{array}\right)\left(\frac{r-2}{r}\right)^{n}, \\
& \mathbf{P}\left(A_{e}^{2}\right) \leqslant \mathbf{P}\left(\tilde{A}_{e}^{2}\right)=r\left(\frac{r-1}{r}\right)^{n}(1-p)^{n} .
\end{aligned}
$$

Утверждение очевидно в силу соотношений (14) и (15).

Лемма 2. Верно неравенство

$$
\mathbf{P}\left(A_{e}^{3}\right) \leqslant \mathbf{P}\left(\tilde{A}_{e}^{3}\right)=(r-1)^{2}\left(\frac{r-2}{r}\right)^{n-1}\left(1-(1-p)^{n}\right) .
$$

Доказательство. Пусть произошло событие $\tilde{A}_{e}^{3}$, и пусть в ребре $e$ не было цвета $\alpha$ в раскраске $\xi$, а в процессе перекраски из него исчез цвет $\beta$. Зафиксируем нумерацию вершин $\sigma$. Тогда в силу (16) найдется вершина $u$ - первая (согласно нумерации $\sigma$ ) вершина в ребре $e$, перекрасившаяся в цвет $\alpha$. Пусть ее номер в ребре $e$ равен $t$. Таким образом, из (16) получаем цепочку соотношений

$$
\begin{aligned}
\mathbf{P}\left(\tilde{A}_{e}^{3} \mid \sigma\right) & \leqslant r(r-1) \sum_{t=1}^{n} \frac{r-1}{r} p\left(\frac{r-2}{r}\right)^{n-t}\left(\frac{1}{r}(r-2) p+\frac{r-2}{r}(1-2 p)\right)^{t-1} \\
& =(r-1)^{2}\left(\frac{r-2}{r}\right)^{n-1} p \sum_{t=1}^{n}(1-p)^{t-1} \\
& =(r-1)^{2}\left(\frac{r-2}{r}\right)^{n-1}\left(1-(1-p)^{n}\right) .
\end{aligned}
$$

В итоге,

$$
\mathbf{P}\left(\tilde{A}_{e}^{3}\right)=\mathbf{E P}\left(\tilde{A}_{e}^{3} \mid \sigma\right) \leqslant(r-1)^{2}\left(\frac{r-2}{r}\right)^{n-1}\left(1-(1-p)^{n}\right) .
$$

Лемма 2 доказана.

Лемма 3. Верно неравенство

$$
\mathbf{P}\left(B_{e, f}\right) \leqslant \mathbf{P}\left(\widetilde{B}_{e, f}\right)=(r-1)^{4}\left(\frac{r-1}{r}\right)^{2 n} p
$$

Доказательство. Пусть

$$
|e \cap f|=h,
$$

a $v$ - произвольная вершина из $e \cap f$. Разобьем событие $\widetilde{B}_{e, f}$ на части в зависимости от 
вершины $v$. Введем событие $\widetilde{B}_{e, f}(v)$ :

$$
\begin{aligned}
& \widetilde{B}_{e, f}(v)=\bigcup_{\alpha, \gamma=1}^{r}\left(\left\{\xi_{v}=\alpha, \eta_{v} \notin\{0, \alpha\}\right\} \cap \bigcap_{s \in f \backslash\{v\}}\left\{\xi_{s} \neq \gamma\right\}\right. \\
& \cap \bigcap_{s \in f \backslash e}\left[\left\{\sigma_{s}<\sigma_{v}, \eta_{s} \neq \gamma\right\} \cup\left\{\sigma_{s}>\sigma_{v}\right\}\right] \\
& \begin{array}{c}
\cap \bigcap_{s \in e}\left[\left\{\sigma_{s}>\sigma_{v}, \xi_{s} \neq \alpha\right\} \cup\left\{\sigma_{s}<\sigma_{v}, \xi_{s} \neq \alpha\right\}\right. \\
\left.\cup\left\{\sigma_{s}<\sigma_{v}, \xi_{s}=\alpha, \eta_{s} \notin\{0, \alpha\}\right\}\right]
\end{array} \\
& \cap \bigcap\left(\left\{\sigma_{s}<\sigma_{v}, \xi_{s} \neq \gamma, \xi_{s} \neq \alpha, \eta_{s} \neq \gamma, \eta_{s} \neq \alpha\right\}\right. \\
& s \in e \cap f \cup\left\{\sigma_{s}<\sigma_{v}, \xi_{s}=\alpha, \eta_{s} \neq \gamma\right\} \\
& \left.\left.\cup\left\{\sigma_{s}>\sigma_{v}, \xi_{s} \neq \gamma, \xi_{s} \neq \alpha\right\}\right)\right\} \text {. }
\end{aligned}
$$

Заметим, что из (17) следует, что

$$
\widetilde{B}_{e, f}=\bigcup_{v \in e \cap f} \tilde{B}_{e, f}(v) .
$$

Введем также

$$
\begin{aligned}
& t=t(v, \sigma)=|\{u \in e \cap f: \sigma(u)>\sigma(v)\}|, \\
& j=j(v, \sigma)=|\{u \in f \backslash e: \sigma(u)<\sigma(v)\}|, \\
& i=i(v, \sigma)=|\{u \in e \backslash f: \sigma(u)<\sigma(v)\}| .
\end{aligned}
$$

Следовательно,

$$
h-1-t=|\{u \in e \cap f: \sigma(u)<\sigma(v)\}| .
$$

Зафиксируем случайную нумерацию $\sigma$. Тогда с учетом новых обозначений из соотношения (18) получаем следующую оценку условной вероятности события $\widetilde{B}_{e, f}(v)$ :

$$
\begin{aligned}
\mathbf{P}\left(\tilde{B}_{e, f}(v) \mid \sigma\right) \leqslant r & (r-1) \frac{1}{r}(r-1) p(1-p)^{j}\left(\frac{r-1}{r}\right)^{n-h} \\
& \times\left(\frac{r-2}{r}(1-2 p)+\frac{1}{r}(r-2) p\right)^{h-t-1}\left(\frac{r-2}{r}\right)^{t} \\
& \times\left(\frac{r-1}{r}\right)^{n-h-i}\left(\frac{r-1}{r}+\frac{1}{r}(r-1) p\right)^{i} \\
= & r(r-1)\left(\frac{r-1}{r}\right)^{2 n-2 h+1}\left(\frac{r-2}{r}\right)^{h-1} \\
& \times p(1-p)^{j}(1-p)^{h-1-t}(1+p)^{i} \\
\leqslant & r(r-1)\left(\frac{r-1}{r}\right)^{2 n-2 h+1}\left(\frac{r-2}{r}\right)^{h-1} p(1-p)^{j}(1+p)^{i} .
\end{aligned}
$$

Последнее неравенство выполнено в силу того, что $(1-p)^{h-1-t}$ оценивается сверху числом 1. Таким образом, в последнем полученном выражении только величины $i=i(\sigma)$, 
$j=j(\sigma)$ зависят от $\sigma$. Следовательно,

$$
\begin{aligned}
\mathbf{P}\left(\widetilde{B}_{e, f}(v)\right) & =\mathbf{E P}\left(\widetilde{B}_{e, f}(v) \mid \sigma\right) \\
& \leqslant r(r-1)\left(\frac{r-1}{r}\right)^{2 n-2 h+1}\left(\frac{r-2}{r}\right)^{h-1} p \mathbf{E}(1-p)^{j(\sigma)}(1+p)^{i(\sigma)} .
\end{aligned}
$$

В силу того, что $\sigma$ имеет равномерное распределение на множестве всех возможных нумераций множества вершин, а ребра $e$ и $f$ имеют одинаковую мощность $n$, величина $\mathbf{E}(1-p)^{j(\sigma)}(1+p)^{i(\sigma)}$ оценивается сверху единицей. Доказательство этого несложного утверждения можно найти, например, в [5], а также в книге [15] (см. §3.2). В итоге, учитывая (19), мы получаем следующую верхнюю оценку вероятности события $\widetilde{B}_{e, f}$ :

$$
\begin{aligned}
\mathbf{P}\left(\widetilde{B}_{e, f}\right) & \leqslant \sum_{v \in e \cap f} \mathbf{P}\left(\widetilde{B}_{e, f}(v)\right) \\
& \leqslant \sum_{v \in e \cap f} r(r-1)\left(\frac{r-1}{r}\right)^{2 n-2 h+1} p\left(\frac{r-2}{r}\right)^{h-1} \\
& =h r(r-1)\left(\frac{r-1}{r}\right)^{2 n-2 h+1} p\left(\frac{r-2}{r}\right)^{h-1} .
\end{aligned}
$$

Легко видеть, что максимум правой части (20) по $h$ достигается при $h=r^{2}-2 r$. Действительно, отношение правых частей при $h$ и $h+1$ равно $h(r-1)^{2} /[(h+1) r(r-2)]$. Данное отношение не превосходит 1 , если $h \leqslant r^{2}-2 r$. Следовательно, максимальное значение оцениваемой вероятности достигается при $h=r^{2}-2 r$. В итоге, для любых ребер $e$ и $f$

$$
\begin{aligned}
\mathbf{P}\left(\widetilde{B}_{e, f}\right) & \leqslant r^{2}(r-1)(r-2)\left(\frac{r-1}{r}\right)^{2 n+1} p\left(\frac{r(r-2)}{(r-1)^{2}}\right)^{r^{2}-2 r} \\
& \leqslant r^{2}(r-1)(r-2)\left(\frac{r-1}{r}\right)^{2 n+1} p \leqslant(r-1)^{4}\left(\frac{r-1}{r}\right)^{2 n} p .
\end{aligned}
$$

Лемма 3 доказана.

\section{4. Завершение доказательства}

Пусть $\mathscr{F}$ - событие, заключающееся в том, что гиперграф $G$ имеет плохое ребро в случайной раскраске $\chi$. Тогда, в силу (13) и лемм 1-3, вероятность этого события оценивается следующим образом:

$$
\begin{aligned}
\mathbf{P}(\mathscr{F})= & \mathbf{P}\left(\bigcup_{e} \mathscr{F}_{e}\right) \leqslant \sum_{e} \mathbf{P}\left(\mathscr{F}_{e}\right) \\
\leqslant & \sum_{e}\left(\mathbf{P}\left(\tilde{A}_{e}^{1}\right)+\mathbf{P}\left(\tilde{A}_{e}^{2}\right)+\mathbf{P}\left(\tilde{A}_{e}^{3}\right)\right)+\sum_{e} \sum_{f \neq e}\left(\mathbf{P}\left(\widetilde{B}_{e, f}\right)\right) \\
\leqslant & |E| \frac{r(r-1)}{2}\left(\frac{r-2}{r}\right)^{n}+|E| r\left(\frac{r-1}{r}\right)^{n}(1-p)^{n} \\
& +|E|(r-1)^{2}\left(\frac{r-2}{r}\right)^{n-1}\left(1-(1-p)^{n}\right) \\
& +|E|^{2}(r-1)^{4}\left(\frac{r-1}{r}\right)^{2 n} p .
\end{aligned}
$$


Нам необходимо подобрать значение параметра $p$ так, чтобы гарантировать выполнение неравенства $\mathbf{P}(\mathscr{F})<1$. В этом случае с положительной вероятностью в случайной раскраске $\chi$ все ребра гиперграфа $G$ будут хорошими и теорема будет доказана.

Возьмем

$$
p=\frac{\ln n}{2 n}
$$

и оценим каждое из слагаемых в правой части (21). Напомним, что по условию $n \geqslant 3$, $r \leqslant n /(2 \ln n)$ и $|E|$ удовлетворяет неравенству (12). Следовательно, первое слагаемое в правой части (21) удовлетворяет соотношению

$$
\begin{aligned}
|E| \frac{r(r-1)}{2}\left(\frac{r-2}{r}\right)^{n} & \leqslant \frac{r(r-1)}{2}\left(\frac{r-2}{r-1}\right)^{n} \frac{1}{2 r}\left(\frac{n}{(r-1)^{2} \ln n}\right)^{1 / 2} \\
& \leqslant \frac{1}{4} e^{-n /(r-1)}\left(\frac{n}{\ln n}\right)^{1 / 2} \leqslant \frac{1}{4} e^{-2 \ln n}\left(\frac{n}{\ln n}\right)^{1 / 2} \\
& =\frac{1}{4\left(n^{3} \ln n\right)^{1 / 2}}<\frac{1}{20}=0,05 .
\end{aligned}
$$

Третье слагаемое оценивается аналогично с помощью (22):

$$
\begin{aligned}
|E|(r-1)^{2}\left(\frac{r-2}{r}\right)^{n-1}\left(1-(1-p)^{n}\right) & \leqslant|E| 2 r(r-1)\left(\frac{r-2}{r}\right)^{n} \\
& \leqslant 4 \cdot 0,05=0,2 .
\end{aligned}
$$

Для второго и четвертого слагаемых справедливы следующие простые неравенства:

$$
\begin{aligned}
|E| r\left(\frac{r-1}{r}\right)^{n}(1-p)^{n} & \leqslant \frac{1}{2}\left(\frac{n}{\ln n}\right)^{1 / 2}(1-p)^{n} \\
& \leqslant \frac{1}{2}\left(\frac{n}{\ln n}\right)^{1 / 2} e^{-p n}=\frac{1}{2(\ln n)^{1 / 2}}<\frac{1}{2}, \\
|E|^{2}(r-1)^{4}\left(\frac{r-1}{r}\right)^{2 n} p & \leqslant \frac{1}{4 r^{2}}\left(\frac{r}{r-1}\right)^{2 n} \frac{n}{(r-1)^{2} \ln n}(r-1)^{4}\left(\frac{r-1}{r}\right)^{2 n} p \\
& \leqslant \frac{1}{4} \frac{n}{\ln n} p=\frac{1}{8} .
\end{aligned}
$$

Таким образом, из неравенств (21), (22), (24), (23) и (25) получаем, что

$$
\mathbf{P}(\mathscr{F})<0,05+0,2+0,5+0,125<1 .
$$

Следовательно, с положительной вероятностью в случайной раскраске $\chi$ все ребра гиперграфа $G$ будут хорошими. Таким образом, для гиперграфа $G$ существует полноцветная раскраска в $r$ цветов. Теорема 1 доказана.

Замечание 1. Отметим, что константа $1 / 2$ в правой части оценки (7) не является оптимальной в асимптотике. В частности, из доказательства теоремы 1 следует, что ее можно заменить выражением, не превосходящим $\sqrt{2}$. Однако нас в первую очередь интересует порядок роста оценки, а не точное вычисление констант при растущих множителях. 


\section{4. Достаточное условие существования} полноцветной $r$-раскраски

Вопрос о существовании полноцветной $r$-раскраски у равномерных гиперграфов можно изучать не только рассматривая ограничения на количество ребер, но и на другие характеристики гиперграфа. Одной из таких характеристик является максимальная степень ребра гиперграфа. Напомним, что степенью ребра $f$ в гиперграфе $H$ называется количество других ребер $H$, которые пересекают $e$. Максимальную степень ребра в гиперграфе $H$ будем обозначать через $\Delta_{e}(H)$.

В 1973 г. в [6] П. Эрдёш и Л. Ловас получили следующее достаточное условие существования полноцветной $r$-раскраски у $n$-равномерного гиперграфа.

Теорема 2 ([6]). Пусть $n, r \geqslant 2$ и $H-n$-равномерныцй гиперграф. Если

$$
\Delta_{e}(H) \leqslant \frac{r^{n-1}}{4(r-1)^{n}},
$$

то для Н сущеествует полноцветная r-раскраска.

Отметим, что доказательство данной теоремы опирается на известное утверждение теории вероятностей, называемое локальной леммой и полученное в той же работе [6].

Позднее результат теоремы 2 был улучшен Шабановым (см. [9]) для достаточно широкой области значений параметра $r$.

Теорема 3 ([9]). Пусть $n, r \geqslant 2 u H-n$-равномерный гиперграф, причем

$$
\Delta_{e}(H) \leqslant \frac{\sqrt{12}-3}{8 r}\left(\frac{n}{(r-1)^{2} \ln n}\right)^{1 / 3}\left(\frac{r}{r-1}\right)^{n} .
$$

Если $r \leqslant n /(8 \ln n)$, то для $Н$ существует полноцветная $r$-раскраска.

Основным результатом настоящего параграфа является следующая теорема, дающая новое достаточное условие существования полноцветной $r$-раскраски гиперграфа в терминах ограничения на максимальную степень вершины в гиперграфе.

Теорема 4. Пусть $n \geqslant 3, r \geqslant 2, u r \leqslant n /(2 \ln n)$. Если $n$-равномерный гиперграф $H$ удовлетворяет условию

$$
\Delta_{e}(H) \leqslant \frac{\sqrt{11}-3}{4 r}\left(\frac{n}{(r-1)^{2} \ln n}\right)^{1 / 2}\left(\frac{r}{r-1}\right)^{n}-1,
$$

то для Н сущзествует полноцветная г-раскраска.

Сравним новый результат с предыдущими. Если $r^{2}=o(n / \ln n)$, то ограничение (27) на $\Delta_{e}(H)$ в теореме 4 , очевидно, асимптотически в $\left(n /\left((r-1)^{2} \ln n\right)\right)^{1 / 6}$ раз лучше ограничения (26) на $\Delta_{e}(H)$ в теореме 3 . В то же время, если $r \gg(n / \ln )^{1 / 2}$, то наша оценка (27) становится асимптотически слабее классического результата Эрдёша и Ловаса.

В заключение отметим, что достаточные условия существования полноцветных раскрасок у равномерных гиперграфов в терминах ограничений на максимальную степень ребра находят применения в абстрактной теории автоматов (подробнее об этом см. [14]). 


\section{1. Доказательство теоремы 4}

Для доказательства нам понадобится классическое утверждение, носящее название локальной леммы. Впервые оно было доказано в работе П. Эрдёша и Л. Ловаса [6]. Мы сформулируем его в специальном виде, удобном для дальнейших рассуждений.

Теорема 5. Пусть на некотором вероятностном пространстве задан набор событий $Q_{1}, \ldots, Q_{N}$. Пусть $S_{1}, \ldots, S_{N}-$ такие подмножества множества $\mathscr{R}_{N}=\{1, \ldots, N\}$, что для любого $i=1, \ldots, N$ событие $Q_{i}$ не зависит от алгебры, порожденной событиями $\left\{Q_{j}: j \in \mathscr{R}_{N} \backslash S_{i}\right\}$. Если для каждого $i=1, \ldots, N$ выполнено неравенство

$$
\sum_{j \in S_{i}} \mathbf{P}\left(Q_{j}\right) \leqslant 1 / 4
$$

mo

$$
\mathbf{P}\left(\bigcap_{j=1}^{N} \bar{Q}_{j}\right)>0
$$

Итак, пусть задан произвольный $n$-равномерный гиперграф $H=(V, E)$, удовлетворяющий условию (27) теоремы 4. Рассмотрим такую же случайную раскраску $\left\{\chi_{s}\right\}_{s \in V}$ для $H$, зависящую от параметра $p$, как и в доказательстве теоремы 1 . Необходимо показать, что для $H$ существует полноцветная $r$-раскраска. Для этого достаточно доказать, что случайная раскраска $\left\{\chi_{s}\right\}_{s \in V}$ будет с положительной вероятностью (при некотором выборе параметра $p$ ) полноцветной $r$-раскраской для $H$.

Введем системы событий

$$
\begin{array}{ll}
\mathscr{A}^{1}=\left\{\tilde{A}_{e}^{1}, e \in E\right\}, & \mathscr{A}^{2}=\left\{\tilde{A}_{e}^{2}, e \in E\right\}, \\
\mathscr{A}^{3}=\left\{\tilde{A}_{e}^{3}, e \in E\right\}, & \mathscr{B}=\left\{\widetilde{B}_{e, f}, e, f \in E\right\},
\end{array}
$$

где события $\tilde{A}_{e}^{1}, \tilde{A}_{e}^{2}, \tilde{A}_{e}^{3}$ и $\widetilde{B}_{e, f}$ были определены в $\$ 3.2$ (см. (14), (15), (16), (17)). В силу соотношений (13), (14), (15), (16), (17),

$$
\left\{\left\{\chi_{s}\right\}_{s \in V} \text { есть полноцветная } r \text {-раскраска для } H\right\} \supset \bigcap_{Q \in A^{1} \cup \mathscr{A}^{2} \cup \mathscr{A}^{3} \cup \Re} \bar{Q} \text {. }
$$

Остается показать, что вероятность последнего события строго больше нуля при некотором выборе параметра $p$. Определим для каждого события $Q \in \mathscr{A}^{1} \cup \mathscr{A}^{2} \cup \mathscr{A}^{3} \cup \mathscr{B}$ такое подмножество событий $S(Q) \subset \mathscr{A}^{1} \cup \mathscr{A}^{2} \cup \mathscr{A}^{3} \cup \mathscr{B}$, что $Q$ не зависит от алгебры, порожденной $\mathscr{A}^{1} \cup \mathscr{A}^{2} \cup \mathscr{A}^{3} \cup \mathscr{B} \backslash S(Q)$, и выполнено условие (28) локальной леммы, которое в наших обозначениях выглядит как

$$
\sum_{\widetilde{Q} \in S(Q)} \mathbf{P}(\tilde{Q}) \leqslant \frac{1}{4}
$$

Необходимо рассмотреть ряд случаев.

Случай 1. Пусть сначала $Q \in \mathscr{A}^{1}$, то есть $Q=\tilde{A}_{e}^{1}$ для некоторого $e \in E$. В силу (14), событие $\tilde{A}_{e}^{1}$ лежит в алгебре, порожденной случайными величинами $\left\{\xi_{s}, \eta_{s}: s \in e\right\}$. Поэтому оно не зависит от всех событий $\tilde{A}_{f}^{1}, \tilde{A}_{f}^{2}, \tilde{A}_{f}^{3}$, если $f \cap e=\varnothing$, и от всех событий 
$\widetilde{B}_{g, f}$ при условии $g \cap e=\varnothing$ и $f \cap e=\varnothing$, а также от алгебры, порожденной этими событиями, потому что данная алгебра лежит в алгебре, порожденной случайной нумерацией $\sigma$ и $\left\{\xi_{s}, \eta_{s}: s \in V \backslash e\right\}$. Таким образом, в качестве $S\left(\tilde{A}_{e}^{1}\right)$ достаточно взять подмножество $\mathscr{A}^{1}$, состоящее из событий $\tilde{A}_{f}^{1}, f \cap e \neq \varnothing$, подмножество $\mathscr{A}^{2}$, состоящее из событий $\tilde{A}_{f}^{2}$, $f \cap e \neq \varnothing$, подмножество $\AA^{3}$, состоящее из событий $\tilde{A}_{f}^{3}, f \cap e \neq \varnothing$, и подмножество $\mathscr{\Re}$, состоящее из событий $\widetilde{B}_{g, f}$ с условием либо $f \cap e \neq \varnothing$, либо $g \cap e \neq \varnothing$. Ясно, что событий первых трех видов не более $\Delta_{e}(H)+1$, а четвертого - не более, чем $2\left(\Delta_{e}(H)+1\right)^{2}$. Таким образом, используя леммы $1-3$, получаем следующую оценку суммы вероятностей:

$$
\begin{aligned}
\sum_{Q \in S\left(A_{e}^{1}\right)} \mathbf{P}(Q) \leqslant & \sum_{f: f \cap e \neq \varnothing} \mathbf{P}\left(\tilde{A}_{f}^{1}\right)+\sum_{f: f \cap e \neq \varnothing} \mathbf{P}\left(\tilde{A}_{f}^{2}\right)+\sum_{f: f \cap e \neq \varnothing} \mathbf{P}\left(\tilde{A}_{f}^{3}\right) \\
& +\sum_{f, g:(f \cap e \neq \varnothing) \cup(g \cap e \neq \varnothing)}\left(\mathbf{P}\left(\widetilde{B}_{f, g}\right)+\mathbf{P}\left(\widetilde{B}_{g, f}\right)\right) \\
\leqslant & \left(\Delta_{e}(H)+1\right)\left[\frac{r(r-1)}{2}\left(\frac{r-2}{r}\right)^{n}+r\left(\frac{r-1}{r}\right)^{n}(1-p)^{n}\right. \\
& \left.+(r-1)^{2}\left(\frac{r-2}{r}\right)^{n-1}\left(1-(1-p)^{n}\right)\right] \\
& +2\left(\Delta_{e}(H)+1\right)^{2}(r-1)^{4}\left(\frac{r-1}{r}\right)^{2 n} p=S_{1} .
\end{aligned}
$$

Выберем параметр $p$ таким же, как в доказательстве теоремы 1:

$$
p=\frac{\ln n}{2 n} .
$$

В силу условия теоремы, что $r \leqslant n /(2 \ln n)$, такой выбор параметра $p$ является корректным: $r p \leqslant 1$. Таким образом, мы можем воспользоваться оценками (22), (23), (24) и (25) из доказательства теоремы 1 (ограничение (27) на $\Delta_{e}(H)+1$ лишь на константу отличается от ограничения (12) на число ребер $|E|$ ). В итоге получаем верхнюю оценку величины $S_{1}$ :

$$
S_{1}<\frac{3}{2} \frac{\sqrt{11}-3}{4}+\left(\frac{\sqrt{11}-3}{4}\right)^{2}<\frac{1}{4} .
$$

Случай $Q \in \mathscr{A}^{2}$ полностью аналогичен случаю $Q \in \mathscr{A}^{1}$.

Случай 2. Пусть $Q \in \mathscr{A}^{3}$, то есть $Q=\tilde{A}_{e}^{3}$ для некоторого $e \in E$. Событие $\tilde{A}_{e}^{3}$ лежит в алгебре, порожденной случайными величинами $\left\{\xi_{s}, \eta_{s}: s \in e\right\}$, а также набором индикаторов $\left\{I\left\{\sigma_{s}<\sigma_{t}\right\}: s, t \in e\right\}$. Поэтому оно не зависит от всех событий $\tilde{A}_{f}^{1}, \tilde{A}_{f}^{2}, \tilde{A}_{f}^{3}$, если $f \cap e=\varnothing$, и от всех событий $\widetilde{B}_{g, f}$ при условии $g \cap e=\varnothing$ и $f \cap e=\varnothing$, а также от алгебры, порожденной этими событиями, потому что данная алгебра лежит в алгебре, порожденной случайными величины $\left\{\xi_{s}, \eta_{s}: s \in V \backslash e\right\}$ и индикаторами $\left\{I\left\{\sigma_{s}<\sigma_{t}\right\}: s, t \in V \backslash e\right\}$. Таким образом, в качестве $S\left(\tilde{A}_{e}^{1}\right)$ достаточно взять подмножество $\mathscr{A}^{1}$, состоящее из событий $\tilde{A}_{f}^{1}$, $f \cap e \neq \varnothing$, подмножество $\mathscr{A}^{2}$, состоящее из событий $\tilde{A}_{f}^{2}, f \cap e \neq \varnothing$, подмножество $\mathscr{A}^{3}$, состоящее из событий $\tilde{A}_{f}^{3}, f \cap e \neq \varnothing$, и подмножество $\mathscr{B}$, состоящее из событий $\widetilde{B}_{g, f}$ с условием либо $f \cap e \neq \varnothing$, либо $g \cap e \neq \varnothing$. По условию теоремы, событий первых 
трех видов не более $\Delta_{e}(H)+1$, а четвертого - не более, чем $2\left(\Delta_{e}(H)+1\right)^{2}$. Используя леммы 1-3, получаем следующую оценку суммы вероятностей:

$$
\begin{aligned}
& \sum_{Q \in S\left(A_{e}\right)} \mathbf{P}(Q) \leqslant \sum_{f: f \cap e \neq \varnothing} \mathbf{P}\left(\tilde{A}_{f}^{1}\right)+\sum_{f: f \cap e \neq \varnothing} \mathbf{P}\left(\tilde{A}_{f}^{2}\right)+\sum_{f: f \cap e \neq \varnothing} \mathbf{P}\left(\tilde{A}_{f}^{3}\right) \\
&+\sum_{f, g:(f \cap e \neq \varnothing) \cup(g \cap e \neq \varnothing)}\left(\mathbf{P}\left(\widetilde{B}_{f, g}\right)+\mathbf{P}\left(\widetilde{B}_{g, f}\right)\right) \\
& \leqslant\left(\Delta_{e}(H)+1\right)\left[\frac{r(r-1)}{2}\left(\frac{r-2}{r}\right)^{n}+r\left(\frac{r-1}{r}\right)^{n}(1-p)^{n}\right. \\
&\left.+(r-1)^{2}\left(\frac{r-2}{r}\right)^{n-1}\left(1-(1-p)^{n}\right)\right] \\
&+2\left(\Delta_{e}(H)+1\right)^{2}(r-1)^{4}\left(\frac{r-1}{r}\right)^{2 n} p=S_{1}<\frac{1}{4} .
\end{aligned}
$$

Последнее неравенство выполнено в силу (29).

Случай 3. Остается разобрать случай, когда $Q \in \mathscr{B}$, то есть $Q=\widetilde{B}_{e, f}$. Из определения события $\widetilde{B}_{e, f}(17)$ следует, что оно лежит в алгебре, порожденной случайными величинами $\left\{\xi_{s}, \eta_{s}: s \in e \cup f\right\}$, а также набором индикаторов $\left\{I\left\{\sigma_{s}<\sigma_{t}\right\}: s, t \in e \cup f\right\}$. Отсюда получаем, что данное событие не зависит от всех событий $\tilde{A}_{g}^{i}$, если $g \cap(e \cup f)=\varnothing$, $i=1, \ldots, 3$, и от всех событий $\widetilde{B}_{g, h}$ при условии $(g \cup h) \cap(e \cap f)=\varnothing$, а также от алгебры, порожденной этими событиями, потому что данная алгебра лежит в алгебре, порожденной случайными величинами $\left\{\xi_{s}, \eta_{s}: s \in V \backslash(e \cup f)\right\}$ и индикаторами $\left\{I\left\{\sigma_{s}<\sigma_{t}\right\}: s, t \in V \backslash(e \cup f)\right\}$. Таким образом, в качестве $S\left(\widetilde{B}_{e, f}\right)$ достаточно взять подмножество $\mathscr{A}^{i}$, состоящее из событий $\tilde{A}_{g}^{i}$ для таких $g$, что $g \cap(e \cup f) \neq \varnothing, i=1, \ldots, 3$, и подмножество $\mathscr{B}$, состоящее из событий $\widetilde{B}_{g, h}$ с условием $(g \cup h) \cap(e \cup f) \neq \varnothing$. По условию теоремы, событий первого типа не больше чем $2\left(\Delta_{e}(H)+1\right)$, второго - не больше чем $4\left(\Delta_{e}(H)+1\right)^{2}$. Как и в первом случае, сумма вероятностей оценивается следующим образом:

$$
\begin{aligned}
& \sum_{Q \in S\left(A_{e}\right)} \mathbf{P}(Q) \leqslant \sum_{g: g \cap(e \cup f) \neq \varnothing} \mathbf{P}\left(\tilde{A}_{g}^{1}\right)+\sum_{g: g \cap(e \cup f) \neq \varnothing} \mathbf{P}\left(\tilde{A}_{g}^{2}\right) \\
&+\sum_{g: g \cap(e \cup f) \neq \varnothing} \mathbf{P}\left(\tilde{A}_{g}^{3}\right)+\sum_{g, h:(g \cup h) \cap(e \cup f) \neq \varnothing}\left(\mathbf{P}\left(\widetilde{B}_{h, g}\right)+\mathbf{P}\left(\widetilde{B}_{g, h}\right)\right) \\
& \leqslant 2\left(\Delta_{e}(H)+1\right)\left[\frac{r(r-1)}{2}\left(\frac{r-2}{r}\right)^{n}+r\left(\frac{r-1}{r}\right)^{n}(1-p)^{n}\right. \\
&\left.+(r-1)^{2}\left(\frac{r-2}{r}\right)^{n-1}\left(1-(1-p)^{n}\right)\right] \\
&+4\left(\Delta_{e}(H)+1\right)^{2}(r-1)^{4}\left(\frac{r-1}{r}\right)^{2 n} p=S_{2} .
\end{aligned}
$$


Используя приведенные ранее оценки (22), (23), (24) и (25), приходим к искомому неравенству:

$$
S_{2}<3 \frac{\sqrt{11}-3}{4}+2\left(\frac{\sqrt{11}-3}{4}\right)^{2}=\frac{1}{4} .
$$

Таким образом, для системы событий $\mathscr{A}^{1} \cup \mathscr{A}^{2} \cup \mathscr{A}^{3} \cup \mathscr{B}$ выполняется условие (28) локальной леммы. Из нее следует, что с положительной вероятностью не случится ни одно из этих событий, поэтому для $H$ существует полноцветная $r$-раскраска. Теорема 4 доказана.

\section{5. Экстремальная задача о степени вершины}

Задача о нахождении величины $p(n, r)$ - это задача о нахождении минимального числа ребер в $n$-равномерном гиперграфе, для которого не существует полноцветной $r$-раскраски. Подобную задачу можно поставить и для других характеристик гиперграфа, например, для максимальной степени вершины. Напомним, что степенью вершины в гиперграфе называется количество ребер этого гиперграфа, которые содержат данную вершину. Будем обозначать максимальную степень вершины в гиперграфе $H$ через $\Delta(H)$.

По аналогии с величиной $p(n, r)$, введем величину $\Delta_{p}(n, r)$, равную минимально возможному значению $\Delta(H)$, где $H-n$-равномерный гиперграф, не имеющий полноцветной $r$-раскраски. Заметим, что из теоремы 4 вытекает следующая нижняя оценка величины $\Delta_{p}(n, r)$.

Следствие 1. Пусть $n \geqslant 3, r \geqslant 2, u r \leqslant n /(2 \ln n)$. Тогда выполняется неравенство

$$
\Delta_{p}(n, r) \geqslant \frac{\sqrt{11}-3}{4 r} \frac{1}{(r-1) \sqrt{n \ln n}}\left(\frac{r}{r-1}\right)^{n} .
$$

Основным результатом данного параграфа является верхняя оценка величины $\Delta_{p}(n, r)$.

Теорема 6. Для любого $\varepsilon>0$ существует такое натуральное число $n_{0}=n_{0}(\varepsilon)$, что для всех $n \geqslant n_{0}$ u $r \geqslant 2$ с условием $r \leqslant \frac{1}{3} n^{2 / 3}$ выполняется неравенство

$$
\Delta_{p}(n, r) \leqslant(1+\varepsilon) \frac{n \ln r}{r} e\left(\frac{r}{r-1}\right)^{n}
$$

Доказательство. Доказательство основано на рассмотрении биномиальной модели случайного гиперграфа. Пусть $K_{N}^{(n)}$ - полный $n$-равномерный гиперграф на $N$ вершинах, тогда случайный гиперграф $H(N, n, p)$ есть подгиперграф $K_{N}^{(n)}$, полученный путем случайного включения ребер $K_{N}^{(n)}$ : каждое ребро независимо от других включается в $H(N, n, p)$ с вероятностью $p$ и не включается с вероятностью $1-p$. Данная модель случайного гиперграфа - это естественное обобщение классической модели случайного графа $G(n, p)$, предложенной в 60-х годах П. Эрдёшем и А. Реньи (см. [16]). Подробнее об исследованиях случайных графов и гиперграфов см. [17, 18, 19].

Оценим вероятность того, что случайный гиперграф $H(N, n, p)$ допускает полноцветную $r$-раскраску. Рассмотрим произвольную раскраску $\sigma_{i}, i=1, \ldots, r^{N}$, множества вершин в $r$ цветов. Пусть $E_{i}$ - соответствующее ей множество плохих ребер, то есть множество ребер $K_{N}^{(n)}$, которые в раскраске $\sigma_{i}$ не содержат вершины хотя бы одного из $r$ 
цветов. Обозначим через $v_{j}(i), j=1, \ldots, r$, количество вершин цвета $j$ в раскраске $\sigma_{i}$. По формуле включения-исключения получаем равенство

$$
\left|E_{i}\right|=\sum_{k=1}^{r-1}(-1)^{r-k+1} S_{k}(i),
$$

где

$$
S_{k}(i)=\sum_{\substack{\left(j_{1}, \ldots, j_{k}\right): \\
1 \leqslant j_{1}<\ldots<j_{k} \leqslant r}}\left(\begin{array}{c}
v_{j_{1}}(i)+\ldots+v_{j_{k}}(i) \\
n
\end{array}\right) .
$$

Легко видеть, что вероятность того, что данная раскраска $\sigma_{i}$ является полноцветной для гиперграфа $H(N, n, p)$ равняется $(1-p)^{\left|E_{i}\right|}$. В [9] было показано, что верно неравенство

$$
\begin{aligned}
\min _{i}\left|E_{i}\right| & =\min _{i} \sum_{k=1}^{r-1}(-1)^{r-k+1} S_{k}(i) \\
& \geqslant r\left(\begin{array}{c}
N-N / r-1 \\
n
\end{array}\right)-\left(\begin{array}{l}
r \\
2
\end{array}\right)\left(\begin{array}{c}
N-2 N / r+2 \\
n
\end{array}\right) .
\end{aligned}
$$

Выберем значение параметра $N$ следующим образом:

$$
N=\left\lceil\frac{n^{2}}{2 r}\right\rceil \text {. }
$$

В [9] было показано, что при таком выборе параметра $N$ и условии $r \leqslant \frac{1}{3} n^{2 / 3}$ правая часть соотношения (32) асимптотически эквивалентна своему первому слагаемому, то есть существует такая функция $\psi_{1}(n)$, не зависящая от $r$ и стремящаяся к 1 с ростом $n$, что

$$
r\left(\begin{array}{c}
N-N / r-1 \\
n
\end{array}\right)-\left(\begin{array}{l}
r \\
2
\end{array}\right)\left(\begin{array}{c}
N-2 N / r+2 \\
n
\end{array}\right) \geqslant r\left(\begin{array}{c}
N-N / r-1 \\
n
\end{array}\right) \psi_{1}(n) .
$$

Следовательно,

$$
\begin{aligned}
& \mathbf{P}(H(N, n, p) \text { не допускает полноцветной } r \text {-раскраски }) \leqslant \sum_{i=1}^{r^{N}}(1-p)^{\left|E_{i}\right|} \\
& \quad \leqslant r^{N}(1-p)^{r\left({ }_{n}^{N-N / r-1}\right) \psi_{1}(n)} \leqslant r^{N} \exp \left\{-p r\left(\begin{array}{c}
N-N / r-1 \\
n
\end{array}\right) \psi_{1}(n)\right\} .
\end{aligned}
$$

Теперь выберем значение параметра $p$. Пусть $\delta_{1}>0$, тогда положим

$$
p=\frac{\left(1+\delta_{1}\right) N \ln r}{r\left(\begin{array}{c}
N-N / r-1 \\
n
\end{array}\right) \psi_{1}(n)}
$$

В силу такого выбора $p$, из (33) получаем итоговую оценку вероятности существования полноцветной $r$-раскраски у $H(N, n, p)$ :

$$
\mathbf{P}(H(N, n, p) \text { допускает полноцветную } r \text {-раскраску }) \leqslant r^{-\delta_{1} N} .
$$


Перейдем к рассмотрению максимальной степени вершины в случайной гиперграфе $H(N, n, p)$. Пусть $v$ - произвольная вершина гиперграфа $H(N, n, p)$. Обозначим через $X_{v}$ ее степень, тогда случайная величина $X_{v}$ имеет биномиальное распределение $\operatorname{Bin}\left(\left(\begin{array}{l}N-1 \\ n-1\end{array}\right), p\right)$. Далее нам понадобится классическая вероятность уклонения биномиальной случайной величины от своего среднего значения: если $\xi-$ биномиальная случайная величина, то для любого $\delta_{2}>0$

$$
\mathbf{P}\left(\xi-\mathbf{E} \xi \geqslant \delta_{2} \mathbf{E} \xi\right) \leqslant e^{-\delta_{2}^{2} \mathbf{E} \xi / 2}
$$

Доказательство этого несложного факта можно найти, например, в книге [18]. Применим данное утверждение к $X_{v}$ :

$$
\mathbf{P}\left(X_{v}-\mathbf{E} X_{v} \geqslant \delta_{2} \mathbf{E} X_{v}\right) \leqslant e^{-\delta_{2}^{2} \mathbf{E} X_{v} / 2} .
$$

Рассмотрим подробнее $\mathbf{E} X_{v}$. С одной стороны, учитывая, что $\psi_{1}(n) \leqslant 1$ и $r \leqslant \frac{1}{3} n^{2 / 3}$, получаем, что

$$
\mathbf{E} X_{v}=\left(\begin{array}{c}
N-1 \\
n-1
\end{array}\right) p=\frac{\left(1+\delta_{1}\right) N(\ln r)\left(\begin{array}{c}
N-1 \\
n-1
\end{array}\right)}{r\left(\begin{array}{c}
N-N / r-1 \\
n
\end{array}\right) \psi_{1}(n)} \geqslant \frac{n \ln r}{r} \geqslant 3 n^{1 / 3} \ln r,
$$

a с другой стороны, в [9] было показано, что при нашем выборе параметра $N=\left\lceil n^{2} / 2 r\right\rceil$ и ограничении $r \leqslant \frac{1}{3} n^{2 / 3}$ выполняется неравенство

$$
\begin{aligned}
\mathbf{E} X_{v} & =\left(\begin{array}{c}
N-1 \\
n-1
\end{array}\right) p=\frac{\left(1+\delta_{1}\right) N(\ln r)\left(\begin{array}{c}
N-1 \\
n-1
\end{array}\right)}{r\left(\begin{array}{c}
N-N / r-1 \\
n
\end{array}\right) \psi_{1}(n)} \\
& \leqslant \frac{\left(1+\delta_{1}\right) n \ln r}{r \psi_{1}(n)} e\left(\frac{r}{r-1}\right)^{n} \psi_{2}(n),
\end{aligned}
$$

где $\psi_{2}(n)-$ некоторая функция, не зависящая от $r$ и стремящаяся к 1 при $n \rightarrow \infty$.

Из неравенств (35), (36) и (37) получаем неравенство

$$
\begin{aligned}
& \mathbf{P}\left(\Delta(H(N, n, p)) \geqslant\left(1+\delta_{2}\right) \frac{\left(1+\delta_{1}\right) n \ln r}{r \psi_{1}(n)} e\left(\frac{r}{r-1}\right)^{n} \psi_{2}(n)\right) \\
& \quad \leqslant N e^{-\delta_{2}^{2} \mathbf{E} X_{v} / 2} \leqslant N r^{-\frac{3}{2} \delta_{2}^{2} n^{1 / 3} / 2}=\left\lceil\frac{n^{2}}{2 r}\right\rceil r^{-\frac{3}{2} \delta_{2}^{2} n^{1 / 3}} .
\end{aligned}
$$

Итак, пусть задано $\varepsilon>0$. Выберем числа $\delta_{1}>0, \delta_{2}>0$ и $n_{0} \in \mathbf{N}$ так, чтобы для любого $n>n_{0}$ и любого $r \geqslant 2$ с условием $r \leqslant \frac{1}{3} n^{2 / 3}$ выполнялись соотношения

$$
\begin{gathered}
\left(1+\delta_{2}\right)\left(1+\delta_{1}\right) \frac{\psi_{2}(n)}{\psi_{1}(n)} \leqslant 1+\varepsilon, \quad r^{-\delta_{1} N}<\frac{1}{3}, \\
\left\lceil\frac{n^{2}}{2 r}\right\rceil r^{-\frac{3}{2} \delta_{2}^{2} n^{1 / 3}}<\frac{1}{3} .
\end{gathered}
$$

Такой выбор параметров, с учетом (34) и (38), гарантирует, что с вероятностью по крайней мере $1 / 3$ случайный гиперграф $H(N, n, p)$ не допускает полноцветных $r$-раскрасок и при этом его максимальная степень вершины удовлетворяет неравенству

$$
\Delta(H(N, n, p)) \leqslant(1+\varepsilon) \frac{n \ln r}{r} e\left(\frac{r}{r-1}\right)^{n},
$$

что и доказывает искомое соотношение (31). Теорема 6 доказана. 
Замечание 2. Отметим, что если отказаться от ограничения $r<\frac{1}{3} n^{2 / 3}$ в условии теоремы 6, то можно обосновать асимптотически более слабую оценку $\Delta_{p}(n, r)$ нежели (31). Рассуждая так же, как и в доказательстве теоремы 6, можно показать, что при всех достаточно больших $n$ и всех $r<n$ выполнено

$$
\Delta_{p}(n, r) \leqslant 2 n \ln r\left(\frac{r}{r-1}\right)^{n} .
$$

\section{Список литературы}

1. Kostochka A. V., On a theorem by Erdős, Rubin and Taylor on choosability of complete bipartite graphs. J. Comb. (2002) 9, №1.

2. Kostochka A. V., Color-critical graphs and hypergraphs with few edges: a survey. In: More Sets, Graphs and Numbers (Győri E., Katona G. O. H., Lovász L., eds.). Springer, Berlin, 2006, pp. 175198.

3. Erdős P., Hajnal A., On a property of families of sets. Acta Math. Acad. Sci. Hungary (1961) 12, №1-2, 87-123.

4. Erdős P., On a combinatorial problem, II. Acta Math. Acad. Sci. Hungary (1964) 15, №3-4, 445-447.

5. Radhakrishnan J., Srinivasan A., Improved bounds and algorithms for hypergraph two-coloring. Random Structures and Algorithms (2000) 16, №1, 4-32.

6. Erdős P., Lovász L., Problems and results on 3-chromatic hypergraphs and some related questions. Colloq. Math. Soc. Janos Bolyai (1973) 10, 609-627.

7. Шабанов Д. А., Об одной комбинаторной задаче Эрдёша. Докл. Акад. наук (2004) 396, №2, 166-169.

8. Шабанов Д. А., Экстремальные задачи для раскрасок равномерных гиперграфов. Изв. РАН. Сер. матем. (2007) 71, №6, 183-222.

9. Шабанов Д. А., О существовании полноцветных раскрасок для равномерных гиперграфов. Матем. сб. (2010) 201, №4, 137-160.

10. Визинг В. Г., Раскраска вершин графа в предписанные цвета. Дискрет. анализ (1976) 29, 3-10.

11. Erdős P., Rubin A. L., Taylor H., Choosability in graphs. Proc. West Coast Conference on Combinatorics, Graph Theory and Computing (1980) 26, 125-157.

12. Alon N., Choice number of graphs: a probabilistic approach. Combinatorics, Probability and Computing (1992) 1, 107-114.

13. Shabanov D. A., On a generalization of Rubin’s theorem. J. Graph Theory (2011) 67, №3, 226-234.

14. Gécseg F., Imreh B., Pluhár A., On the existence of finite isomorphic complete systems of automata. J. Automata, Languages and Combinatorics (1998) 3, №2, 77-84.

15. Alon N., Spencer J. H., The probabilistic method. Wiley, New York, 1992.

16. Erdős P., Rényi A., On the evolution of random graphs. Magyar Tud. Akad. Mat. Kutató Int. Közl. (1960) 5, №1-2, 17-61.

17. Bollobás B., Random graphs. Cambridge University Press, Cambridge, 2001.

18. Janson S., Luczak T., Ruciński A., Random graphs. Wiley, New York, 2000.

19. Karoński M., Luczak T., Random hypergraphs. Bolyai Soc. Math. Stud. (1996) 2, 283-293.

Статья поступила 9.09.2011. 